\title{
Geomagnetic field declination: from decadal to centennial scales
}

\author{
Venera Dobrica $^{1}$, Crisan Demetrescu ${ }^{1}$, and Mioara Mandea $^{2}$ \\ ${ }^{1}$ Institute of Geodynamics, Romanian Academy, Bucharest, Romania \\ ${ }^{2}$ Centre National d'Etudes Spatiales, Paris, France \\ Correspondence: Venera Dobrica (venera@geodin.ro) \\ Received: 24 October 2017 - Discussion started: 8 November 2017 \\ Revised: 20 February 2018 - Accepted: 24 March 2018 - Published: 25 April 2018
}

\begin{abstract}
Declination annual mean time series longer than 1 century provided by 24 geomagnetic observatories worldwide, together with 5 Western European reconstructed declination series over the last 4 centuries, have been analyzed in terms of the frequency constituents of the secular variation at inter-decadal and sub-centennial timescales of 20-35 and 70-90 years. Observatory and reconstructed time series have been processed by several types of filtering, namely Hodrick-Prescott, running averages, and Butterworth. The Hodrick-Prescott filtering allows us to separate a quasioscillation at a decadal timescale, which is assumed to be related to external variations and called the "11-year constituent", from a long-term trend. The latter has been decomposed into two other oscillations called "inter-decadal" and "sub-centennial" constituents by applying a Butterworth filtering with cutoffs at 30 and 73 years, respectively. The analysis shows that the generally accepted geomagnetic jerks occur around extrema in the time derivative of the trend and coincide with extrema in the time derivative of the 11-year constituent. The sub-centennial constituent is traced back to 1600 in the five 400-year-long time series and seems to be a major constituent of the secular variation, geomagnetic jerks included.
\end{abstract}

\section{Introduction}

The temporal variation of the geomagnetic field has been monitored for decades, mainly by continuous recordings in geomagnetic observatories. In spite of their growing number, their geographical coverage is highly uneven. Since the longest recorded series of observations at geomagnetic observatories do not exceed some 150 years, research has seen increasing interest in historical spot measurements to con- struct time series of geomagnetic elements (declination and inclination) going back over centuries as long as possible (Malin and Bullard, 1981, for the London area; Cafarella et al., 1992a, b, for Rome; Barraclough, 1995, for Edinburgh; Alexandrescu et al., 1996, 1997; Mandea and Le Mouël, 2016, for the Paris area; Korte et al., 2009, for the Munich area). Korte et al. (2009) also included archeomagnetic data in order to infer information going back to AD 1400. Such an interest has also been present in Eastern Europe (Bucha, 1959, for the Czech and Slovak territories; Valach et al., 2004, for Slovakia; Atanasiu, 1968; Constantinescu, 1979; Soare et al., 1998, for Romania); reconstructions go back to 1850 and include intensity elements of the geomagnetic field. Also note the collection of declination and inclination data from sea voyages over the 15-18th centuries by Jonkers et al. (2003) and the spherical harmonics (SHs) model by Jackson et al. (2000). This model includes these data and describes the geomagnetic field evolution since 1590 for all geomagnetic elements (the intensity values are based on an assumed uniform dipole decay rate before 1850).

The last 4 decades - since the finding by Courtillot et al. (1978) of "geomagnetic jerks" - have seen a research focus on the features of the time evolution of the geomagnetic field originating in the Earth's core (main field). Geomagnetic jerks are viewed phenomenologically as sharp changes, within 1 year or so, in the temporal variation of the main field (secular variation) expressed as the first time derivative of the geomagnetic field time series or steps in the secular acceleration expressed as the second time derivative. The definition of geomagnetic jerks is usually illustrated by declination $(D)$ or by the eastward horizontal component of the field $(Y)$ thought to be the least influenced by external variations. Alexandrescu et al. (1997) and Korte et al. (2009) explored the possibility that jerks also occurred before the modern era 
for which they were evidenced. A review on the subject has been published by Mandea et al. (2010). The geomagnetic jerks have been treated as chaotic fluctuations of the field by Qamili et al. (2013). Brown et al. (2013) have researched the sharp time changes in the secular variation using an improved method to account for the external variations in the data (Wardinski and Holme, 2011) and for a jerk identification (Pinheiro et al., 2011); they find by far more events than had previously been determined, with a general trend of an increased number of identified jerks towards the end of the 20 th century and the beginning of the 21 st century. The new satellite era offers high-resolution magnetic data and the possibility to deeply investigate these events. Indeed, Finlay et al. (2015) and Chulliat et al. (2015) indicate the occurrence, in 2006, 2009, and 2012, of geomagnetic secular acceleration pulses at the core surface. We can note as the dates of these new events 2003 (Olsen and Mandea, 2007), 2007 (Olsen et al., 2009; Chulliat et al., 2010), 2011 (Chulliat and Maus, 2014), and 2014 (Torta et al., 2015; Kotzé, 2017).

Periodicities have been found in the evolution of the geomagnetic field. Fourier spectral analysis (e.g., Currie, 1973; Alldredge, 1977; Langel et al., 1986), the maximum entropy method (Jin and Thomas, 1977), empirical mode decomposition (Roberts et al., 2007; Jackson and Mound, 2010), and calculations of torsional waves in the Earth's core (Zatman and Bloxham, 1997; Dickey and de Viron, 2009; Buffett et al., 2009) have pointed to periodicities at several timescales, such as $\sim 11,13-30,50-60$, and 60-90 years. Buffett (2014) explores the MAC (magnetic, Archimedes, and Coriolis forces) waves and their role in generating longperiod variations (60 years) of the field. Periods shorter than the solar cycle timescale have also been detected, both in some geomagnetic field models (Silva et al., 2012) and in observatory data (e.g., Ou et al., 2017). These studies revealed periodicities in the time domain of about 4-7 years and 23 years. The latter was considered of external origin, while the former has been used as an argument in favor of a 6-year internal variation. Recent studies by Gillet et al. (2010, 2015) and Holme and de Viron (2013) point to a $\sim 6$-year variation originating in the core that is also observable in length-ofday data. The signal was also discussed by Abarca del Rio et al. (2000), Mound and Buffet (2006), Cox et al. (2016), and Duan et al. (2018). We note, however, that both periodicities are very close to the harmonics of the solar cycle, suggesting a possible external origin.

By analyzing the frequency content of the geomagnetic field variability, De Santis et al. (2003) and Lesur et al. (2018) have been able to reveal the behavior of the geomagnetic field as either chaotic or stochastic. The former showed that the temporal spectra of the geomagnetic field at the Earth's surface could be approximated for the 1871-2000 time span by a power law with a negative slope of about -4 (in the frequency range corresponding to periods from 7 to 64 years). The latter showed, in shorter time series covering the time interval 1957-2014 and in their model coefficients, that in the case of SV the corresponding power-law slope is about -2 , which is in accordance with the De Santis et al. (2003) results and with the Gillet et al. (2013) hypothesis in deriving the main field COV-OBS model. Also, Lesur et al. (2018) succeeded in reaching periods down to 1 or 2 years.

Demetrescu and Dobrica (2014) demonstrate the presence, in 24 observatory time series (annual means of the $D, H$, $Z$ components over some 100-150 years), of constituents of secular variation at timescales of 22 and $\sim 80$ years superimposed on a so-called steady variation. A slightly different timescale, of 70-75 years and not of $\sim 80$ years as seen in $H$ and $Z$ data, is characteristic of declination. They show that these constituents are also seen in the first time derivative of the field. The episodes of increasing and decreasing secular variation that result from the superposition of the two constituents are separated by a smooth transition that lasts several years; the sharpness of a geomagnetic jerk is decided by the external effects still existing in the data. Indeed, the external effects, mainly controlled by the 11-year solar cycle, are still present when averaging available data to obtain the annual means. These remaining external contributions are present mostly in the intensity elements of the recorded field $(H, Z)$ and affect the declination less (e.g., Olsen and Mandea, 2007). The effects of external contributions in studying the secular variation have been emphasized and quantitatively shown for European observatories by Verbanac et al. (2007), in terms of correcting annual means using information on external sources, by Wardinski and Holme (2011) in terms of a stochastic (covariant) modeling method and by Dobrica et al. (2013) in terms of secular variation maps. Demetrescu and Dobrica (2014) tentatively show that the $\sim 80$-year variation can be traced back to the 15 th century using three long time series of declination for London (Malin and Bullard, 1981), Rome (Cafarella et al., 1992a, b), and Munich (Korte et al., 2009).

In the present paper we focus on declination data and revisit 24 time series of observatory data by updating the available measurements with additional ones since 2007; the last year is included in a previous analysis (Demetrescu and Dobrica, 2014). Additional data allow us to better constrain the 1999 (Mandea et al., 2000) and 2007 (Chulliat et al., 2010) events and to infer a possible external contribution to the 2003 geomagnetic jerk (Olsen and Mandea, 2007). Here, new methods are applied in filtering the time series and novel approaches regarding the quasi-periodicities of the constituents at longer timescales are considered (Hodrick and Prescott, 1997). Additionally, special attention is given to the 11-year solar-cycle-related constituent present in the declination annual means. Finally, we elaborate on our previous analysis of three very long declination time series by (i) including two more for Paris (Alexandrescu et al., 1996, 1997; Mandea and Le Mouël, 2016) and Edinburgh (Barraclough, 1995), (ii) discussing the first time derivative of the five time series, (iii) comparing in detail our analysis on jerk occurrence to the Alexandrescu et al. (1997) and Korte et al. (2009) ones, 


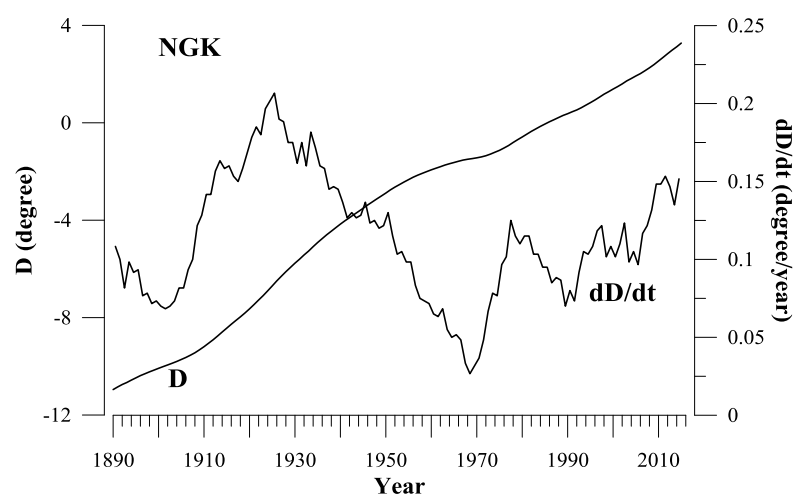

Figure 1. Declination and its first time-derivative time series. Example for a high-standards geomagnetic observatory (Niemegk, NGK).

and (iv) comparing with time series provided by the $\mathrm{gufm} I$ main field model by Jackson et al. (2000).

\section{Data}

\subsection{Observatory data}

Annual means of declination as given by http://www. geomag.bgs.ac.uk/data_service/data/annual_means.shtml have been used. The locations of the 24 observatories with 100-150-year-long time series, labeled with their IAGA codes, are shown in Fig. S1 (Supplement) superimposed on the WMM2010 declination map at the geomagnetic epoch 2010.0 (http://www.ngdc.noaa.gov/geomag/WMM/data/ WMM2010/WMM2010_D_MERC.pdf). In Fig. 1 we show, as an example, time series of declination and of its first time derivative at Niemegk (Germany) Observatory (IAGA code NGK).

\subsection{Historical data}

The five long time series referenced above for Edinburgh, London, Paris, Munich, and Rome have been considered in the present study. Data used, in the order of decreasing latitude of the location, are as follows.

- Edinburgh: raw data published by Barraclough (1995), adjusted to Eskdalemuir Observatory (ESK);

- London: raw data published by Malin and Bullard (1981), adjusted to Hartland Observatory (HAD);

- Paris: raw data published by Alexandrescu et al. (1996, 1997) and reviewed recently by Mandea and Le Mouël (2016), adjusted to Chambon-la-Forêt Observatory (CLF);
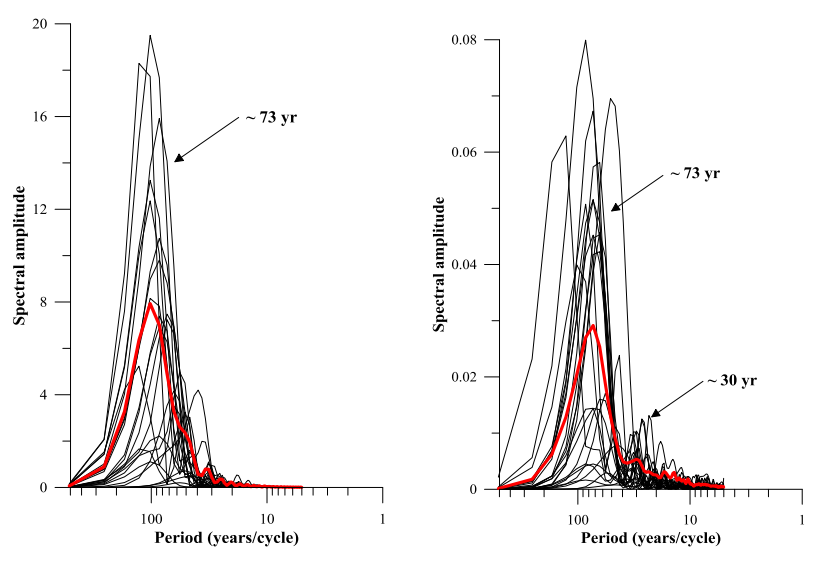

(a)

Figure 2. FFT power spectrum: observatory declination time series (a) and time derivative of declination time series (b). The average power spectrum (red).

- Munich: 11-year filtered smoothing spline fitted to raw data, as published by Korte et al. (2009), adjusted to Fürstenfeldbruck Observatory (FUR);

- Rome: assembled time series using data published by Cafarella et al. (1992b) for the Rome area and for three successively operating Italian observatories (Pola, 1881-1922; Castellaccio, 1933-1962; L'Aquila, 19602011), adjusted to L'Aquila Observatory (AQU).

These time series are used in Sect. 4.

\section{Method}

Fourier spectral analyses (FFT) of the 24 declination time series (Fig. 2a) and of their time derivative (Fig. 2b), done on detrended time series by removing a straight line fit from data, show a broad spectral peak at around 73 years $(60$ 100 ) that dominates by far other (broad) peaks at $\sim 30, \sim 22$, and $\sim 17$ years. It is worth mentioning that these periods are in good agreement with those found by Jackson and Mound (2010) using a different approach, empirical mode decomposition, on declination and inclination time series from 48 geomagnetic observatories (the periods found by these authors being $11.5,30.5,62$, and 81 years). We also show the power spectra of declination and of its secular variation in a log-log scale, following De Santis et al. (2003) and Lesur et al. (2018), in Fig. S2 (Supplement). The end-toend straight line was removed from the time series before the FFT analysis to avoid end effects. While the average power spectrum of declination time series might be represented by a power law with an exponent of -3.7 in the frequency range corresponding to periods from 100 to 7 years, the average power spectrum of secular variation shows a slope of -1.88 (for periods of 100-10 years). These figures confirm the re- 
sults of De Santis et al. (2003) and Lesur et al. (2018) regarding the slope of the power law describing the FFT log$\log$ plots in the case of the field and its secular variation. A change in slope occurring at a period of about 10 years can be noticed in the case of SV. Figure S2 (bottom plot) indicates "lines", or rather, periods grouped at three timescales, namely $60-90,20-35$, and $8-15$ years, exactly those we considered for our filtering approach. At odds with the loglog plot, which is expected to smooth the spectral lines, the power spectral density versus frequency or period in a semilog scale plot is able to comparatively show the magnitude of various oscillations present in the time series.

At this stage we also note differences between observatories regarding frequencies corresponding to these lines, which are commented upon below. Some of these differences could arise from the different lengths of the time series, as some tests (not shown here) of repeating the FFT for the same time series truncated to different lengths seem to indicate. Demetrescu and Dobrica (2014) notice that dominating powerful signals at larger timescales in the data tend to contaminate the filtered time series meant to show quasi-periodic variations at smaller timescales. That is why in the present paper we apply a Hodrick and Prescott (1997; HP) analysis, which is able to separate oscillatory features at smaller (e.g., decadal) timescales from trends representing variations at larger (e.g., centennial) timescales.

The HP filter separates a time series $y_{t}$ into a trend component $T_{t}$ and a cyclical component $C_{t}$ such that $y_{t}=T_{t}+C_{t}$. The function for the filter has the form

$\sum_{t=1}^{m} C_{t}^{2}+\lambda \sum_{t=1}^{m}\left[\left(T_{t}-T_{t-1}\right)-\left(T_{t-1}-T_{t-2)}\right]^{2}\right.$,

where $m$ is the number of samples and $\lambda$ is the smoothing parameter. The first sum minimizes the difference between the time series and its trend component (which is its cyclical component). The second sum minimizes the second-order difference in the trend component (which is analogous to minimization of the second derivative of the trend component). If the smoothing parameter is 0 , no smoothing takes place. As the smoothing parameter increases in value, the smoothed series becomes more linear. Appropriate values of the smoothing parameter depend upon the data sampling. In our case with the data being sampled yearly we apply a smoothing parameter of 100, as recommended by Hodrick and Prescott (1997) and checked by us after a few tests with $\lambda$ varying between 10 and 1600 with a step of 50 (not shown here). According to Paige and Trindade (2010), the HP filter is a special case of a linear penalized spline model with knots placed at all observed time points.

Variations at larger timescales seen in the trend given by HP filtering have been further decomposed into two other oscillations by applying a Butterworth (1930) filtering with certain cutoffs corresponding to periods of $\sim 73$ and 30 years, as indicated by two broad peaks in the superimposed FFT

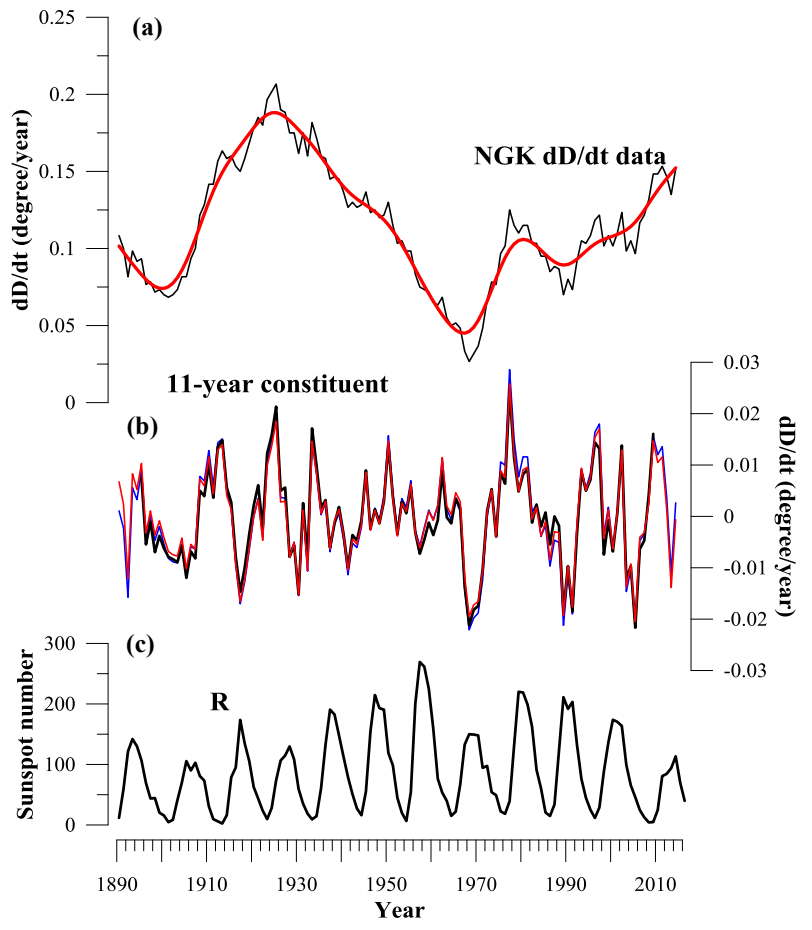

Figure 3. Constituents of the first time derivative of the declination at Niemegk Observatory. (a) First differences in annual means (thin black curve) and the trend from an HP filtering (thick red curve). (b) Cyclic constituent from an HP filtering (red), the 11-year signal from filtered first differences by an 11-year cutoff high-pass Butterworth filtering (blue), and the 11-year signal obtained as a residual after the removal of an 11-year running average from the $d \mathrm{D} / d \mathrm{t}$ time series (black). (c) The sunspot number time series $(R)$.

spectra of the trend for all 24 observatories shown in Fig. S3. The above cutoffs are derived as averages over the 24 FFT spectral lines, as Silva et al. (2012), Demetrescu and Dobrica (2014), and Ou et al. (2017) did with their data. It is important to note that no matter what figure was used in our filter design (except the actual period in the data), the filtered time series would show the actual oscillations hidden in the unfiltered time series (Demetrescu and Dobrica, 2014, Appendix).

\subsection{Observatory data}

We have applied the described methods on observatory and historical data. With respect to observatory data, firstly we show in Fig. 3, as an example of data processing, results for Niemegk. The first time derivative of declination (the first differences in annual means) is shown in Fig. 3a, together with the trend given by the HP filter. The cyclic component is also plotted (panel b). No difference exists when the latter is compared to the superimposed time series obtained as residuals of filtering the original time series with an 11-year running average window or with a high-pass 11 -year cutoff 
Butterworth (1930) filter. Verbanac et al. (2007) show that the residual signal after removing the CM4 core field model from the annual averages of European observatories has a clear solar cycle signature and can be modeled down to $\pm 2 \mathrm{nT}$ using magnetospheric ring current data (Dst) and an ionospheric field proxy (Ap). Wardinski and Holme (2011) characterize the external effects stochastically by analyzing the correlated so-called "noise" in the time series of $X, Y$, and $Z$ components at worldwide-distributed observatories and the Dst index in a first step and residuals at Niemegk instead of Ap in a second step. Here, we also mention attempts at extracting external contributions through global models on long periods (Yukutake and Cain, 1979; McLeod et al., 1996; Langel et al., 1996; Gillet et al., 2013), which give an idea of what can be achieved on the basis of spherical harmonics decomposition, and of the expected respective amplitudes of internal and external signals. We tend to attribute the 11-year signal to external sources based on a long list of papers referenced by Demetrescu and Dobrica (2014). In Fig. 3c, the sunspot number time series have been plotted as an independent indication of the external origin of the cyclic component. It might be seen that the cyclic component is almost in opposite phase with the 11-year solar cycle, but more detailed aspects will be addressed in a future study. In a recent review (Finlay et al., 2017), it is once more underlined that external field characterization is a challenge for main geomagnetic field modeling. We are aware, however, that our point of view regarding the signal at these frequencies as external could be considered partially correct, as new models based on satellite data succeed in reducing the ambiguity in the internalexternal source separation. We note that core sources could also contribute to the high-frequency 11-year signal and its constituents, for instance by the 6-year signal discussed by Gillet et al. (2010, 2015) and Holme and de Viron (2013).

Since the HP filtering applied to the trend is not able to further separate it into constituents, we apply (a) running averages (Demetrescu and Dobrica, 2014) and (b) Butterworth (1930) filtering to get the time series corresponding to the $\sim 73$ - and at 25-35-year timescales. In Fig. 4 we show, again as an example, the results for NGK. In the first case, the constituents of the trend are obtained by successively smoothing the trend time series with a 30- and 73-year running average and subtracting them from the trend time series and from the 30-year average time series. A similar result is obtained in the second case of using 30- and 73-year cutoff Butterworth filtering. Both methods have advantages and disadvantages. As one can notice in Fig. 4, the running average filtering produces time series that include the full information from the unfiltered data on a certain central portion, but no information for both ends of the time series (the 30- and the 73-year smoothed time series are shorter by 15 and 36 years, respectively, at each end), while the Butterworth filter produces time series of the same length as unfiltered data, but with distorted amplitudes at the ends. The dates of maxima and minima in the filtered time series are, however, correctly

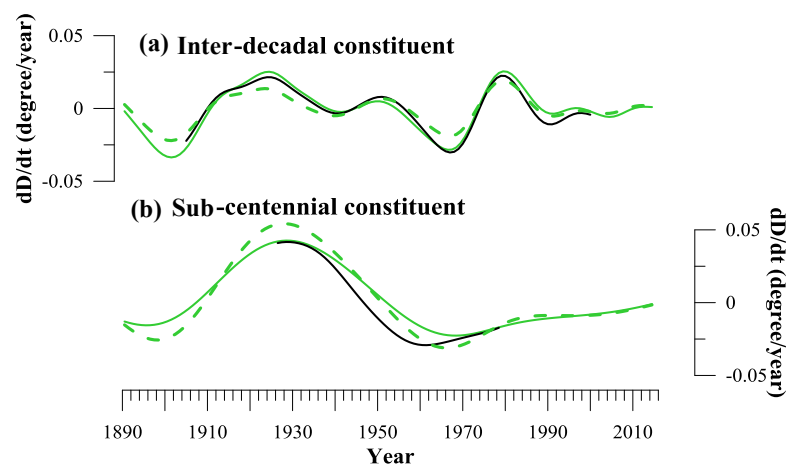

Figure 4. Constituents of the first time-derivative trend of declination at Niemegk Observatory (the red curve in Fig. 3a). (a) The inter-decadal constituent of the trend as a 30-year cutoff high-pass Butterworth filtering (green) and as a residual after the removal of a 30 -year running average filtering (black) from the trend, the " $\sim 22$ year" constituent from a 22-year cutoff high-pass Butterworth filtering (dashed green). (b) The sub-centennial constituent of the trend as a 73-year cutoff Butterworth filtering (green) and as a residual after the removal of a 73-year running average filtering from the 30-year smoothed trend (black), the " 80-year" constituent from a 78-year cutoff Butterworth filtering (dashed green).

retrieved, allowing conclusions on (quasi-)periodicities in the data to be drawn for the entire time interval covered. Note that if we have used instead of 30- and 73-year filtering on trend values the 22- and 80-year filtering by Demetrescu and Dobrica (2014), we would have obtained similar results (extrema at the same moments, but slightly different amplitudes), as one can see in Fig. 4. Of course, any pair of constituents one chooses, whether 30- and 73- or 22- and 80-year ones, the sum of the two constituents is the same, namely the trend plotted in Fig. 3a.

Let us discuss the differences between observatories seen in frequencies corresponding to the broad spectral lines singled out above (Figs. 2 and S3). The information regarding the actual periodicities at various observatories would not be lost when adopting a certain average value (e.g., 73 or 80 years) in the data processing. Indeed, unless the window in the running average or the cutoff value in the Butterworth filtering is a multiple of the hidden period in the data, the filtered cyclical component is itself a cyclical component of the same period as the original component (Appendix in Demetrescu and Dobrica, 2014). This can be seen in Fig. 4, in which time series obtained using values of 22 and 30 years or 80 and 73 years for filtering data are compared. In the case of $H$ and $Z$ (not shown) the filtered $\sim 80$-year constituent shows an oscillation of that period (actually a mean period of 78 years), but in the case of $D$ a slightly different timescale of 70-75 years seems to be characteristic. Since $D$ is a nonlinear function of the field vector, one should not expect the field oscillations induced by the core sources to be identical in $H$ and $D$ (Roberts et al., 2007), so a slight difference 
might occur. Also, one should not expect exactly the same response at all observatories to variations in the core sources changes. Consequently, in the remainder of this paper we use the terms "inter-decadal" and "sub-centennial" constituents for the ones at the 20-35 and 70-90-year timescales, respectively.

We remark here that, at odds with the internal interdecadal and sub-centennial constituents, the 11-year constituent is very noisy, on the one hand because errors in the annual means (measurement noise, baseline definition, changes in pillars, etc.) are retained almost entirely in this time series and on the other hand as a result of the timederivative operator that enhances noise and brings forward the harmonics of the 11-year constituent that are not significant in the data (compare also Fig. 2a and b). The solar cycle length variability (between 8 and 14 years over the past 10 cycles) also contributes to the noise in the high-passed 11-year time series. By superimposing spectra of the cyclic component for the 24 declination time series (Fig. S4), the noisiness is evident. However, in spite of that, some specific lines can be distinguished:

- lines in the 15-25-year interval corresponding to the $\sim 22$-year constituent detected in the last $\sim 40$ years of the cyclic component time series of Fig. 3;

- lines in the 8-14-year domain corresponding to the 11year constituent; and

- lines in the 4-7- and 2-3-year domains corresponding to the first two harmonics of the 11-year constituent (we note that the 4-7-year signal covers the 6-year signal detected in variations of length of day (Holme and de Viron, 2013) and in wave processes discussed by Gillet et al. $(2010,2015)$, pointing to a possible core contribution to the observed variation. We also note the presence of stronger peaks in the 2-3-year period domain than those in the 4-7-year one. This observation is in line with the study by Ou et al., 2017).

We are aware of the fact that, due to noise, the separation in frequency domains is not an ideal one. For instance, there are large peaks occurring between frequencies corresponding to periods between 7 and 9 years; they characterize spectra for Hartland and, with smaller amplitude, Canberra Observatory. We consider this as possibly linked to the less precise values at the beginning of the time series, with noise that is retained in the 11-year signal and can significantly alter the FFT.

\subsection{Historical data}

The historical data have been processed as has been done for observatory time series. Since data are sparser and sparser before $\sim 1800$, for London, Paris, Rome, and Edinburgh a cubic B-spline interpolation of early data is used to obtain a plot with continuous annual values, after removing the evident outliers. The latter can be a result of less precise mea-

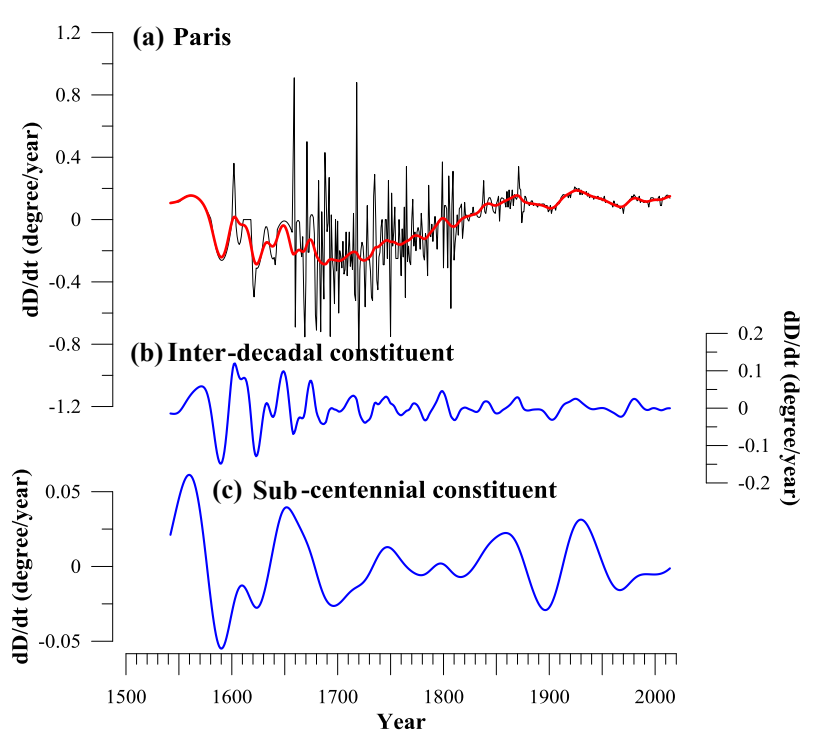

Figure 5. The first time derivative of the Paris declination time series (black) and the HP trend (red) (a); the inter-decadal (b) and the sub-centennial (c) constituents of the trend from a Butterworth filtering.

surements and/or less precise reduction to the location of the present-day observatory contributing with data over the most recent periods. Due to the temporal distance of several years between historical data at the beginning of the reconstructed time series, the spline line might show artifact wiggles. For that part of the time series a linear or quadratic interpolation could be more appropriate. The Munich series has already been filtered by Korte et al. (2009).

Figure 5 shows the time derivative of the Paris time series together with the superimposed HP trend; the inter-decadal and sub-centennial constituents of the trend are also shown. The noise problem becomes stronger when the time derivative of the declination series is considered. The time derivative enhances, as expected, short time variations presented at decadal or shorter timescales by less accurate historical data before $\sim 1850$; this results in higher amplitudes than for the observatory era of the two constituents of the HP trend (compare 0.12 to 0.025 and 0.05 to 0.01 in the case of the inter-decadal and sub-centennial constituents, respectively).

\section{Results and discussion}

\subsection{Observatory data}

The two constituents of the secular variation for the 24 observatories considered in this study as obtained by an HP filtering, namely the trend and the decadal cyclic variations, are shown in Fig. 6. The trends are referred to as the average value for the time interval in which they are defined. The two constituents of the trend, the inter-decadal and the sub- 


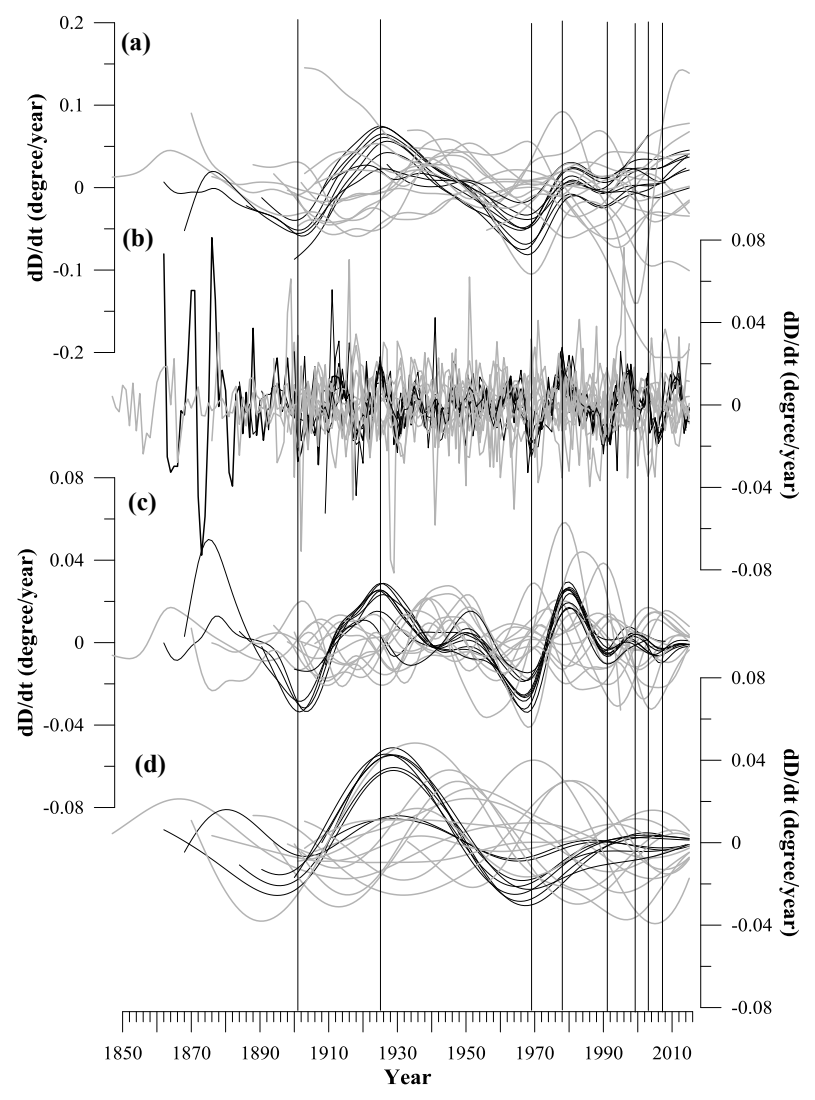

Figure 6. Constituents of the first time derivative of the declination at all analyzed observatories (European time series in black and non-European in gray). From (a) to (d): the trend from an HP filtering, the cyclical constituent from an HP filtering, the inter-decadal constituent of the trend, and the sub-centennial constituent of the trend. Vertical lines mark the generally accepted 20th century geomagnetic jerks.

centennial variations as obtained by a Butterworth filtering, are also plotted. We superimpose the time series from the 24 observatories corresponding to each of the timescales in order to emphasize common features and differences. We also indicate in Fig. 6 the accepted occurrence time of geomagnetic jerks (e.g., Mandea et al., 2010; Brown et al., 2013).

The cyclic constituent obtained from an HP filtering (Fig. 6b) is assumed to show the effects of external sources in the data; it is very noisy and prevents in this form any interpretation regarding this constituent of the recorded secular variation. However, plotting only data from the considered European observatories (Fig. 7) emphasizes the strong presence of the harmonics of the 11-year cycle superimposed on the 11-year oscillations in the first part of the time series and on the significant 22-year oscillation in the last $\sim 40$ years. We have chosen geographically close European observatories in order to enhance the visual effect, as they are affected by the same 22 - and $\sim 80$-year variations (see Demetrescu

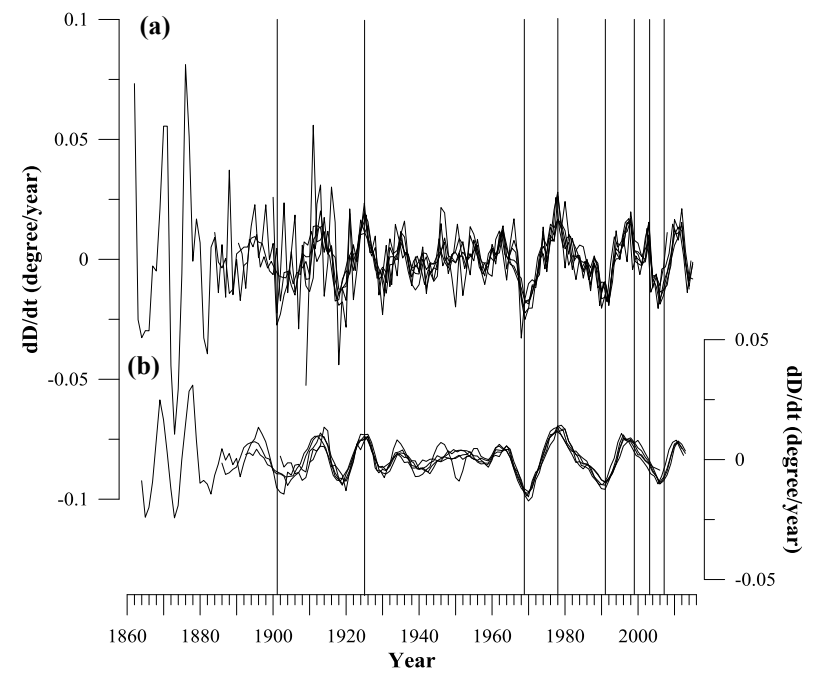

Figure 7. (a) The decadal variation of the first time derivative of declination at the European observatories (cyclical constituent from an HP filtering). (b) The 5-year running average smoothing of the time series plotted in (a). Vertical lines mark the generally accepted 20th century geomagnetic jerks.

and Dobrica, 2014) and show similar time evolutions. The similarity of the 11-year signal in European observatories is also noticed in a previous paper (Dobrica et al., 2013).

The 2003 jerk shown in Fig. 6 has been evidenced for limited areas only $\left(\Delta \mathrm{d} Y^{2} / \mathrm{d} t^{2}\right.$ slightly negative over Central and Eastern Europe and positive along the $90-100^{\circ} \mathrm{E}$ meridian; noted by Olsen and Mandea, 2007). From Fig. 7 it seems that the external effects might play a role in characterizing the 2003 jerk, as a 5-year running average smoothing meant to get rid of variations related to the first harmonic of the 11-year variation attenuates the sharp variation of the declination time derivative seen in the raw data (Fig. 7a). We note that the smoothing would also attenuate the 6-year component discussed by Gillet et al. $(2010,2015)$ and Holme and de Viron (2013) if present in the data. According to the same figure, other recent European geomagnetic jerk occurrence dates should be slightly shifted by 1 year, from 1999 and 2007 to 1998 and 2006, respectively, when extrema actually occur in the 11-year variation. However, due to the filtering procedure and the annual mean sampling of the data, the events by the end of the series might not be so well described. It is worth noting that in terms of the analysis shown in Figs. 6 and 7, the very recent geomagnetic jerks that occurred in 2011 and 2014, as evidenced for limited areas by Chulliat and Maus (2014) and Torta et al. (2015) in the Atlantic sector and the Atlantic and European sectors, respectively, reveal a strong influence of the decadal constituent. A more detailed analysis of the 11-year constituent of the secular variation is beyond the scope of this paper.

In Figs. 6 and 7 the vertical lines mark epochs of accepted geomagnetic jerks; they occurred around extrema in the time 
derivative of the trend variation, produced by a combination of the two constituents at $\sim 30$ and $\sim 73$-year timescales, and coincide with extrema in the time derivative of the external variation (with the above observation regarding the 1999 and 2007 events). Phase differences between individual time series explain differences in the occurrence time and geographical distribution of geomagnetic jerks. Once the external contributions to the first differences in the observatory annual means of comparable amplitude with the trend variations are minimized, the core contribution to the observed secular variation no longer exhibits the very sharp appearance of geomagnetic jerks. According to our quantitative analysis of recorded data presented in Figs. 6 and 7 (see also Demetrescu and Dobrica, 2014), the geomagnetic jerk might be seen as a result of a more general phenomenon, namely the evolution of the secular variation as a result of a superposition of two (or several) waves describing the effects of processes in the Earth's core at two (or several) timescales. This is in line with Bloxham et al. (2002) and Alldredge $(1984,1985)$, who advanced this possibility based on core flow modeling arguments and on geometric arguments, respectively. Finlay and Jackson (2003) and Jackson and Finlay (2007) have identified core surface equatorial westward-moving magnetic flux patches that can be either a result of core flow vortices entrained by a larger-scale westward flow or Alfvén waves excited in the core. That the flow in the core is turbulent became a common consideration with many studies on geodynamo and core flow modeling from secular variation data (see the review by Holme, 2015, on the latter). The turbulent flow, as inferred by De Santis et al. (2003) by looking at the power spectra of $X, Y$, and $Z$ annual means time series, does not exclude but rather implies the existence of vortices with various timescales and space scales. The "waves" we speak of above could in fact be surface manifestations of core surface vortices that move around and survive for a given time span, as shown by Demetrescu and Dobrica (2014). The latter discussed the map appearance of their steady 22-year and $\sim 80$-year variations, pointing to the different space scales of the three ingredients that manifest themselves at the three timescales. As noted by Holme (2015), the core flow models existing to date, including those of higher resolution based on satellite models of the field and secular variation, are not able to predict small-scale features, as field models cannot resolve details in the core field smaller than the spherical harmonic degree 13.

\subsection{Historical data}

Demetrescu and Dobrica (2014) have previously analyzed three of the long time series of historical magnetic declination data (London, Munich, Rome) and showed that the sub-centennial variation is present back in time to the 15 th century. Here, we define and characterize the sub-centennial variation in the case of the secular variation of declination for five available time series.

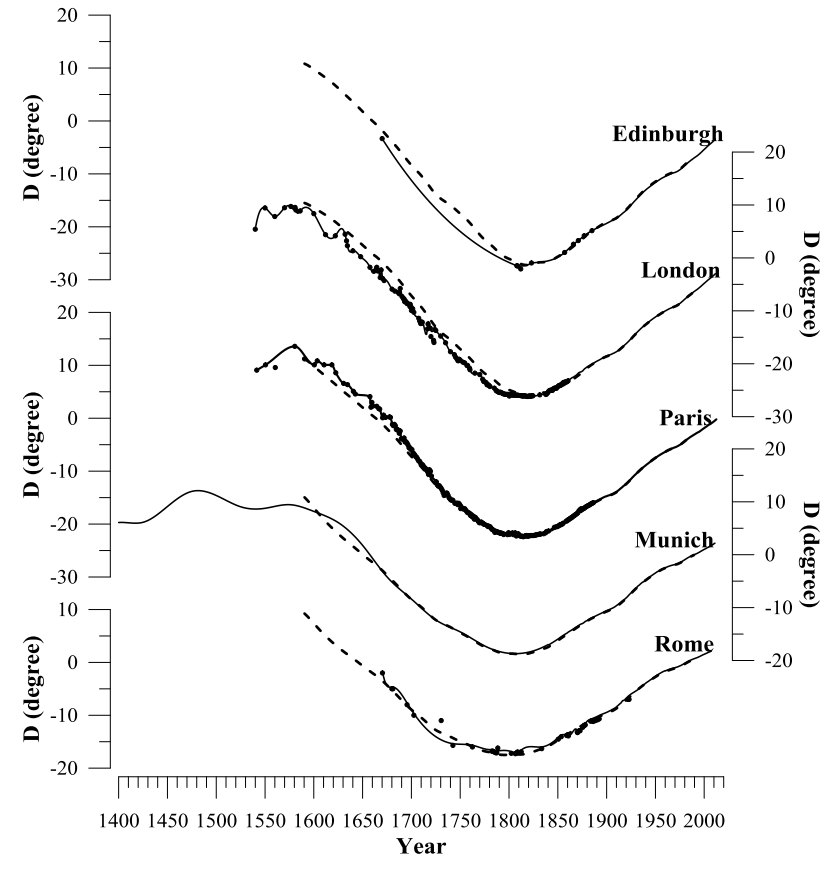

Figure 8. The declination evolution since AD 1400 at five locations in Europe. Annual values as obtained by a cubic B-spline interpolation on historical observations and observatory data (full black curve) and as obtained from the gufml time series (dashed curve).

The time series showing the declination at the five locations are plotted in Fig. 8. We also superimpose time series obtained from gufml (Jackson et al., 2000) for the corresponding location. The spot measurements before the observatory era are affected by much larger errors than observatory measurements due on the one hand to the equipment accuracy and on the other to the non-corrected external variations and/or reduction to the location of the present-day observatory. As mentioned in Sect. 2, for London, Paris, Rome, and Edinburgh a cubic B-spline interpolation of early data is used to obtain a plot with continuous annual values. The spline curve shows artifact wiggles before $\sim 1700$. For that part of time series a linear or quadratic interpolation could be more appropriate to describe the long-term evolution. The Munich time series published by Korte et al. (2009) had been smoothed with an 11-year filter and a 2.5-year knot space spline. Figure 9 shows, for the five locations, only the first time derivative of the HP trend together with the corresponding HP trend time derivative in $g u f m l$ values.

In the following, only the sub-centennial constituent, which is less affected by noise than the decadal and interdecadal constituents, is discussed. The five curves in Fig. 10, showing the sub-centennial constituent of the trend plotted in Fig. 9, demonstrate that the latter is not restricted to the last 150 years. So does the sub-centennial constituent derived from gufml. The same conclusion based on empirical mode decomposition applied to the Munich data has been invoked 


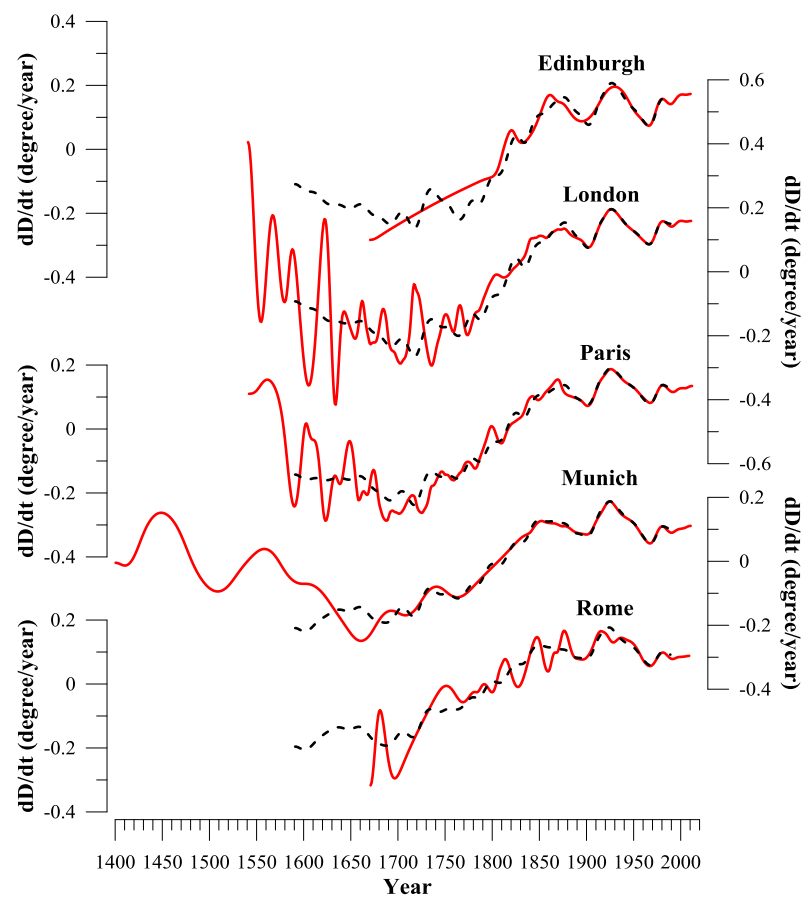

Figure 9. The first time derivative of the HP trend in the data (red) and in the $g u f m l$ (dashed black) for the five locations in Europe.

by Jackson and Mound (2010). The latter also found a longer period of 160 years. Referring to the appearance of the curves in Figs. 8 and 9, a variation at a much larger timescale of 400 years or longer could, however, be present in the data.

Several maxima and minima are evident in the subcentennial constituent time-derivative plots (Fig. 10) before 1900 , in addition to the known ones over the 20th century. By comparing the five time series of the sub-centennial constituent time derivatives with each other and with the corresponding gufml model, a few interesting observations can be emphasized.

Firstly, all curves as derived from the data and/or model show the same maxima and minima of the sub-centennial constituent after 1850 , namely maxima at $1850-1880$ and 1920-1930 and minima at around 1900 and 1960; however, the sub-centennial signal is noisier at the beginning of the five time series and mismatches to gufml are evident at 1650 and 1700 for Munich and Rome, while the gufml time series are consistent with each other. Taking into account the noise in the historical data (Fig. 9) and the fact that $g u f m l$ is based on a much larger number of measurements, not only on the five time series considered here, the gufml is conceivably able to satisfactorily represent the sub-centennial variation at the beginning of the time series.

Secondly, the amplitude of the sub-centennial constituent before and after 1900 seems to be comparable, in spite of the lower quality of the data for the first 300 years of the time series. Larger-amplitude variations at the start of the time se-

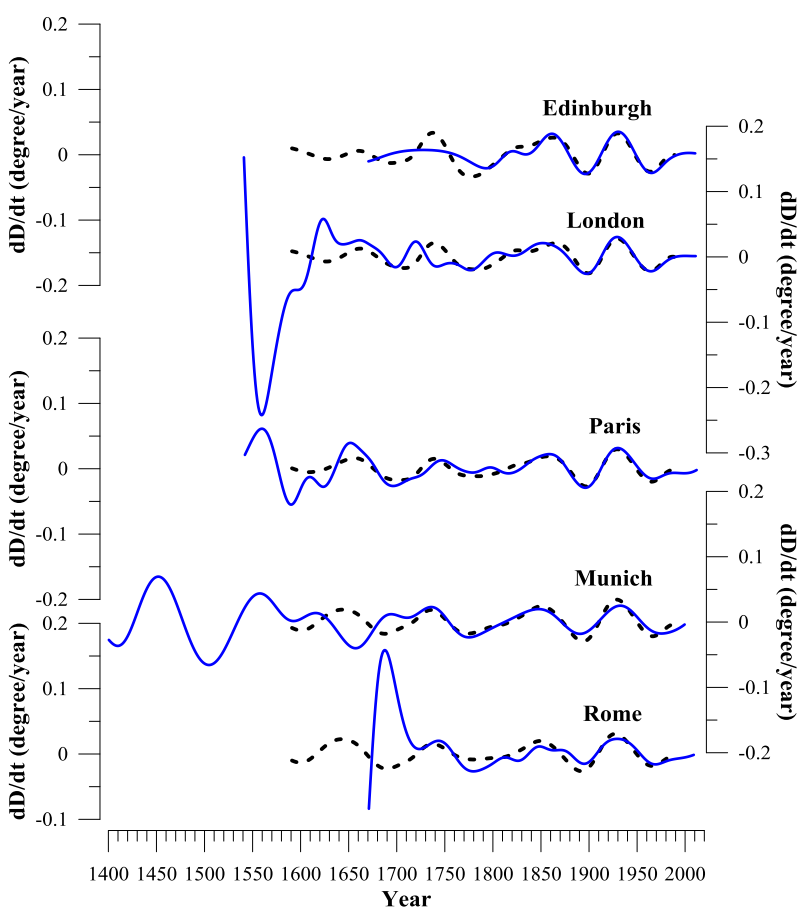

Figure 10. The first time derivative of the sub-centennial constituent at the five locations from the trend of observed data (blue) and of gufml (dashed black); a high-pass 73-year cutoff Butterworth filtering on the time series in Fig. 9 is applied.

ries probably stem from the poorly constrained data in that time interval.

Finally, before 1850 the noise in the data is more evident in the case of the London time series. However, a synchronous maximum around 1800 and a minimum around 1820 can be seen in the London, Paris, and Rome curves, but not in the Munich and in the gufml ones that show only an inflexion at $1790-1800$ and at $\sim 1820$ (change to a lower secular acceleration - the second time derivative of the field - and then change again to a higher secular acceleration). Another maximum that is well developed around 1750 in the Paris, Munich, and Rome plots, as well as in all gufml ones is not seen in the London curve. Back toward 1650, another maximum seems to be present in the London and Paris curves, but not in the Munich one, while the gufml plots indicate its presence. We might attribute this behavior to the temporally less dense data before 1780 and to the spline smoothing. Again, gufml, which is based on a much larger set of measurements, shows consistent behavior among the five locations considered and could support the existence of the sub-centennial variation all the way back to 1600 .

In terms of geomagnetic jerks, Alexandrescu et al. (1997), based on a synthetic declination curve for Paris inferred from the Paris and London series, recognized one event around 1870 in annual and monthly means time series, also detected in Helsinki, Fürstenfeldbruck, and Oslo data. Other possi- 
ble events are noted around 1700, 1730, 1750, 1770, and 1785. These dates correspond within a decade to the maxima and minima of the sub-centennial variation time derivative plotted in Fig. 10 (1690, 1740, 1780, 1800, 1850-1860, 1900), which is satisfactory given the uncertainty on both sides of this comparison. Data prior to 1870 were considered by Alexandrescu et al. (1997) as too noisy or unreliable to clearly reveal geomagnetic jerks at least of comparable amplitude with the 1870, 1901, 1925, 1969, and 1978 jerks. However, as mentioned above, the amplitude of the subcentennial variation before and after 1900 is comparable. Korte et al. (2009) compared the Munich smoothed secular variation time series with a time series of the same length (14002000) for Paris that contains archeomagnetic and other measured data prior to 1700 , is adjusted to CLF, and is splinesmoothed in the same way as the Munich time series. A good agreement characterizes the time interval 1770-2000 and, as the authors stated, surprisingly consistent secular variation and acceleration between the smoothed curves from the two locations is found for the time span 1400-1580, in spite of the rather low quality of the data over this time span. Significant differences between the two locations exist, however, between 1580 and 1770. Korte et al. (2009) also note that in both curves the time interval 1765-1865 seems to be devoid of strong rapid secular changes.

When comparing the possible geomagnetic jerks (called "events" by Korte et al., 2009) in the Munich curve with the maxima and minima of the first time derivative of the sub-centennial constituent for the same location plotted in Fig. 10, we find that most of them $(1448,1508,1558,1693$, $1741,1861,1889,1932)$ coincide within $0-3$ years with the maxima and minima of our sub-centennial constituent (1460, $1510,1560,1690,1740,1780,1800,1850-1860,1900)$. In the time interval 1765-1865, considered to be devoid of strong rapid secular changes by Korte et al. (2009), our analysis detects, as mentioned above, inflections at 1790-1800 and at $\sim 1820$, which are close to the 1790-1810 maximum and to the 1818-1828 minimum seen in the London, Paris, and Rome curves.

We note that the similar variability shown by the field at the five European locations is not surprising, as the on one hand a core source with Earth-surface effects on a large area was active the whole timespan of the model (Stefan et al., 2017). On the other hand, the spatial resolution of the gufml, which decreases significantly towards the start of the model, could also contribute.

Considering these results we can suggest that geomagnetic jerks are only part of a variation at a much longer timescale: the sub-centennial constituent. A certain contribution, most visible over the last 40 years, also comes from the 20-30year inter-decadal constituent. The larger noise in the data before 1900 prevents a possible identification of the latter at earlier times, and the only variation that could be observed is sub-centennial.

\section{Conclusions}

Our results underline the importance of the time perspective one has on geomagnetic data: besides the contribution of the sub-centennial constituent in defining geomagnetic jerks, what we called "steady variation" based on 150 years of observatory data (Demetrescu and Dobrica, 2014) proves to be only part of a larger timescale variation when 400 years of data are available.

Declination annual means time series longer than 1 century provided by 24 geomagnetic observatories worldwide, together with 5 reconstructed declination series over the last 4 centuries in Western Europe, have been analyzed in terms of the frequency constituents of the secular variation at inter-decadal and sub-centennial timescales of 20-35 and 70-80 years. Observatory time series until 2015 have been processed by several types of filtering, namely HodrickPrescott, running averages, and Butterworth. Average windows and cutoffs at 11, 30, and 73 years have been used to account for broad lines in the FFT spectra corresponding to (a) the external solar-cycle-related contamination in the annual averages, the so-called 11-year or decadal constituent, (b) a 20-35-year constituent named inter-decadal, and (c) a broad intense spectral line (60-100 years) present in the data, the so-called sub-centennial constituent, singled out by the HP filtering and FFT analysis of the constituents. The average values used in filtering to obtain the variations of the three constituents of the observed time derivative of declination have no consequences on the evolution and dominant period of the retrieved constituents at individual observatories. This is expected, as any filtered cyclical component is itself a cyclical component of the same period as the original one. Also, a slight difference between average subcentennial timescales in declination and in the vector components of the field could be noticed (73 years compared to 78 years). These results confirm the conclusion by Demetrescu and Dobrica (2014) based on shorter sub-centennial and inter-decadal time series.

The accepted geomagnetic jerks occur around more pronounced extrema in the time derivative of the inter-decadal constituent and coincide with extrema in the time derivative of the 11-year constituent (except the 1999 and the 2007 events). Around 1925, 1969, and 2006, the extrema in the sub-centennial constituent coincide in time or are close to the extrema in the inter-decadal constituent, leading to more pronounced geomagnetic jerks. Phase differences between individual time series explain differences in the occurrence time, geographical distribution, and magnitude of geomagnetic jerks. Once the external contributions to the first differences in the observatory annual means - of comparable amplitude with the inter-decadal and sub-centennial constituents - are minimized, the observed secular variation no longer exhibits a clear V-shape at the time of geomagnetic jerks. We are aware, however, that in doing so, some possibly impor- 
tant internal signal could have also been removed, and further work is necessary to elucidate this problem.

The detected extrema in the historical data have been compared with events interpreted in terms of geomagnetic jerk occurrence dates proposed by other authors (Alexandrescu et al., 1997; Korte et al., 2009). It appears that possible "events" in jerk terms, at 1700, 1730, 1750, 1770, and 1785, considered with a question mark by Alexandrescu et al. (1997) because of too-noisy or unreliable data, are occurring close to the maxima and minima of the sub-centennial constituent. The sub-centennial constituent has comparable amplitudes before and after 1900, in spite of the lower quality of the data in the first 300 years of the analyzed time series, making it a reliable tracer of geomagnetic jerks in the past. Unfortunately, because of noise in the reconstructed time series, the inter-decadal variation, a constituent of the secular variation, could not be recovered and complete information on these phenomena is limited.

According to our results, epochs of geomagnetic jerks may vary as much as a couple of years from one series to another. Over the investigated period, some very clear long periods exist between two successive and well-defined jerks. Over this long-term tendency, less well-defined events can be observed, such as the many noted since magnetic satellite data have been available (e.g., Torta et al., 2015). We suggest that the geomagnetic jerk concept should be considered as a more general notion, namely the evolution of the secular variation as a result of the superposition of two (or more) constituents describing the effects of processes in the Earth's core at two (or more) timescales. Revealing the causes of these variations from the point of view of mechanisms in the core is beyond the scope of this work.

Data availability. The data used in this paper are freely available at World Data Center of Geomagnetism, Edinburgh, http://www. geomag.bgs.ac.uk/data_service/data/annual_means.shtml (last access: 19 April 2018).

\section{The Supplement related to this article is available online at https://doi.org/10.5194/se-9-491-2018-supplement.}

Competing interests. The authors declare that they have no conflict of interest.

Acknowledgements. The historical geomagnetic declination has been compiled from papers by the following authors: Stuart R. C. Malin and Edward C. Bullard; Lili Cafarella, Angelo De Santis, and Antonio Meloni; David R. Barraclough, Mioara Alexandrescu, Vincent Courtillot, and Jean-Louis Le Mouël; and Monika Korte, Mioara Mandea, and Jurgen Matzka (op cit). The study has been carried out in the framework of the project IDEI-UEFISCDI 93/2011. Partial results were presented at MagNetE5 (Rome, 2011) and IUGG (Melbourne, 2011) meetings. We are indebted to Lili Cafarella (INGV-Rome), who kindly provided historical geomagnetic data for Italy, and to the anonymous geomagnetic observatory staff and the World Data Centers on Geomagnetism for obtaining and keeping the data used in this study. We also thank Susan Macmillan, Jon Mound, and the topical editor, Nicolas Gillet, for constructive remarks that improved the paper.

Edited by: Nicolas Gillet

Reviewed by: Jon Mound and Susan Macmillan

\section{References}

Abarco del Rio, R., Gambis, D., and Salstein, D. A.: Interannual signals in length of day and atmospheric angular momentum, Ann. Geophys., 18, 347-364, https://doi.org/10.1007/s00585000-0347-9, 2000.

Alexandrescu, M., Courtillot, V., and Le Mouël, J. -L.: Geomagnetic field direction in Paris since the mid-sixteenth century, Phys. Earth Planet. Inter., 98, 321-360, 1996.

Alexandrescu, M., Courtillot, V., and Le Mouël, J. -L.: High resolution secular variation of geomagnetic field in Western Europe over the last 4 centuries: comparison and integration of historical data from Paris and London, J. Geophys. Res., 102, 2024520258, 1997.

Alldredge, L. R.: Geomagnetic variations with periods from 13 to 30 years, J. Geomagn. Geoelectr., 29, 123-135, 1977.

Alldredge, L. R.: A discussion of impulses and jerks in the geomagnetic field, J. Geophys. Res., 86, 7957-7965, 1984.

Alldredge, L. R.: More on the alleged 1970 geomagnetic jerk, Phys. Earth Planet. Inter., 39, 255-264, 1985.

Atanasiu, G.: Variation séculaire des éléments géomagnétiques en Transylvanie et au Banat (Roumanie) durant la période 18481968, Rev. Roum. Géol., Géophys., Géogr., Serie de Géophysique, 12, 87-104, 1968.

Barraclough, D. R.: Observations of the Earth's magnetic field made in Edinburgh from 1670 to the present day, Trans. R. Soc. Edinburgh: Earth Sci, 85, 239-252, 1995.

Bloxham, J., Zatman, S., and Dumberry, M.: The origin of geomagnetic jerks, Nature, 420, 65-68, 2002.

Brown, W. J., Mound, J. E., and Livermore, P. W.: Jerks abound: An analysis of geomagnetic observatory data from 1957 to 2008, Phys. Earth Planet. Int., https://doi.org/10.1016/j.pepi.2013.06.001, 2013.

Bucha, V.: Säkular Variation der Verticalcomponent Z, Erdmagnetische Feldes fur Zeitraum 1850-1950 in der Tschechoslovakai, Geophys. Sbornik, 114, 281-300, 1959.

Buffett, B.: Geomagnetic fluctuations reveal stable stratification at the top of the Earth's core, Nature, 507, 484-487, https://doi.org/10.1038/nature13122, 2014.

Buffett, B., Mound, J., and Jackson, A.: Inversion of torsional oscillations for the structure and dynamics of Earth's core, Geophys. J. Int., 177, 878-890, https://doi.org/10.1111/j.1365246X.2009.04129.x, 2009.

Butterworth, S.: On the theory of filter amplifiers, Wireless Engineer, 7, 536-541, 1930. 
Cafarella, L., De Santis, A., and Meloni, A.: Secular variation in Italy from historical geomagnetic field measurements, Phys. Earth Planet. Int., 73, 206-221, 1992a.

Cafarella, L., De Santis, A., and Meloni, A.: Il catalogo geomagnetico storico italiano, 160 pp., Istituto Nazionale di Geofisica, Roma, $1992 b$.

Chulliat, A. and Maus, S.: Geomagnetic secular acceleration, jerks, and a localized standing wave at the core surface from 2000 to 2010, J. Geophys. Res.-Solid Earth, 119, 1531-1543, https://doi.org/10.1002/2013JB010604, 2014.

Chulliat, A., Thébault, E., and Hulot, G.: Core field acceleration pulse as a common cause of the 2003 and 2007 geomagnetic jerks, Geophys. Res. Lett., 37, L07301, https://doi.org/10.1029/2009GL042019, 2010.

Chulliat, A., Alken, P., and Maus, S.: Fast equatorial waves propagating at the top of the Earth's Core, Geophys. Res. Lett., 42, 3321-3329, https://doi.org/10.1002/2015GL064067, 2015.

Constantinescu, L.: Early geomagnetic information concerning the Romanian territory, Mem. Sect. Sti., Serie IV, 2, 147-165, 1979.

Courtillot, V., Ducruix, J., and Le Mouël, J.-L.: Sur une accélération récente de la variation séculaire du champ magnétique terrestre, C. R. Hebd. Séances Acad. Sci. Paris, Sér. D, 287, 1095-1098, 1978.

Cox, G. A., Livermore, P. W., and Mound, J. E.: The observational signature of modeled torsional waves and comparison to geomagnetic jerks, Phys. Earth Planet. Int., 255, 50-65, https://doi.org/10.1016/j.pepi.2016.03.012, 2016.

Currie, R. G.: Geomagnetic line spectra -2 to 70 years, Astrophys. Space Sci., 21, 425-438, 1973.

Demetrescu, C. and Dobrica, V.: Multi-decadal ingredients of the secular variation of the geomagnetic field. Insights from long time series of observatory data, Phys. Earth Planet. Inter., 231, 39-55, https://doi.org/10.1016/j.pepi.2014.03.001, 2014.

De Santis, A., Baraclough, D. R., and Tozzi, R.: Spatial and temporal spectra of the geomagnetic field and their scaling properties, Phys. Earth Planet. Int., 135, 125-134, https://doi.org/10.1016/S0031-9201(02)00211-X, 2003.

Dickey, J. O. and de Viron, O.: Leading modes of torsional oscillations within the Earth's core, Geophys. Res. Lett., 36, L15302, https://doi.org/10.1029/2009GL038386, 2009.

Dobrica, V., Demetrescu, C., and Stefan, C.: Toward a better representation of the secular variation. Case study: the European network of geomagnetic observatories, Earth Planet. Space, 65, 767-779, https://doi.org/10.5047/eps.2012.12.001, 2013.

Duan, P., Liu, G., Hu, X., Zhao, J., and Huang, C.: Mechanism of the interannual oscillation in length of day and its constraint on the electromagnetic coupling at the core-mantle boundary, Earth Planet. Sci. Lett., 482, 245-252, 2018.

Finlay, C. C. and Jackson, A.: Equatorially dominated magnetic field change at the surface of the Earth's core, Science, 300, 2084-2086, 2003.

Finlay, C. C., Olsen, N., and Tøffner-Clausen, L.: DTU candidate field models for IGRF-12 and the CHAOS-5 geomagnetic field model, Earth Planet. Space, 67, 114, https://doi.org/10.1186/s40623-015-0274-3, 2015.

Finlay, C. C., Lesur, V., Thébault, E., Vervelidou, F., Morschhauser, A., and Shore, R.: Challenges handling magnetospheric and ionospheric signals in internal geomagnetic field modelling,
Space Sci. Rev., 206, 157-189, https://doi.org/10.1007/s11214016-0285-9, 2017.

Gillet, N., Jault, D., Canet, E., and Fournier, A.: Fast torsional waves and strong magnetic field within the Earth's core, Nature, 465, 74-77, https://doi.org/10.1038/nature09010, 2010.

Gillet, N., Jault, D., Finlay, C. C., and Olsen, N.: Stochastic modeling of the Earth's magnetic field: Inversion for covariances over the observatory era, Geochem. Geophys. Geosyst., 14, 766-786, https://doi.org/10.1002/ggge.20041, 2013.

Gillet, N., Jault, D., and Finlay, C. C.: Planetary gyre, timedependent eddies, torsional waves, and equatorial jets at the Earth's core surface, J. Geophys. Res.-Solid Earth, 120, 39914013, https://doi.org/10.1002/2014JB011786, 2015.

Hodrick, R. J. and Prescott, E. C.: Postwar U. S. business cycles: An empirical investigation, J. Money, Credit, Banking, 29, 1-16, 1997.

Holme, R.: Large-scale flow in the core, in: Treatise on Geophysics, edited by: Schubert, G. and Olson, P., Vol. 8. Core Dynamics, 2nd Eedition, Elsevier, Amsterdam, 91-113, 2015.

Holme, R. and de Viron, O.: Characterization and implications of intradecadal variations in length of day, Nature, 499, 202-204, https://doi.org/10.1038/nature12282, 2013.

Jackson, A. and Finlay, C. C.: Geomagnetic secular variation and its application to the core, in: Treatise on Geophysics, edited by: Schubert, G. and Kono, M., Vol. 5. Geomagnetism, Elsevier, Amsterdam, 147-193, 2007.

Jackson, A., Jonkers, A. R. T., and Walker, M. R.: Four centuries of geomagnetic secular variation from historical records, Phil. Trans. R. Soc. Lond., 358, 957-990, 2000.

Jackson, L. P. and Mound, J. E.: Geomagnetic variations on decadal time scales: What can we learn from Empirical Mode Decomposition?, Geophys. Res. Lett., 37, L14307, https://doi.org/10.1029/2010GL043455, 2010.

Jin, R. and Thomas, D.: Spectral line similarity in geomagnetic dipole field variations and length of day fluctuations, J. Geophys. Res., 82, 828-834, 1977.

Jonkers, A. R. T., Jackson, A., and Murray, A.: Four centuries of geomagnetic data from historical records, Rev. Geophys., 41, 1006, https://doi.org/10.1029/2002RG000115, 2003.

Korte, M., Mandea, M., and Matzka, J.: A historical declination curve for Munich from different data sources, Phys. Earth Planet. Int., 177, 161-172, 2009.

Kotzé, P. B.: The 2014 geomagnetic jerk as observed by southern African magnetic observatories, Earth Planet. Space, 69, 17, https://doi.org/10.1186/s40623-017-0605-7, 2017.

Langel, R. A., Kerridge, D. J., Barraclough, D. R., and Malin, S. R. C.: Geomagnetic temporal change: 1903-1982, A spline representation, J. Geomag. Geoelectr., 38, 573-597, 1986.

Langel, R. A., Sabaka, T. J., Baldwin, R. T., and Conrad, J. A.: The near-Earth magnetic field from magnetospheric and quiet-day ionospheric sources and how it is modelled, Phys. Earth Planet. Int., 98, 235-267, 1996.

Lesur, V., Wardinski, I., Baerenzung, J., and Holschneider, M.: On the frequency spectra of the core magnetic field Gauss coefficients, Phys. Earth Planet. Int., 276, 145-158, https://doi.org/10.1016/j.pepi.2017.05.017, 2018.

Malin, S. R. C. and Bullard, E. C.: The direction of the Earth's magnetic field at London, 1570-1975, Philos. Trans. R. Soc. London, 299, 357-423, 1981. 
Mandea, M. and Le Mouël, J.-L.: After some 350 years - zero declination again in Paris, Hist. Geo Space Sci., 7, 73-77, 2016.

Mandea, M., Bellanger, E., and Le Mouël, J.-L.: A geomagnetic jerk for the end of 20th century? Earth Planet Sci. Lett., 183, 369-373, 2000.

Mandea, M., Holme, R., Pais, A., Pinheiro, K., Jackson, A., and Verbanac, G.: Geomagnetic jerks: Rapid core field variations and core dynamics, Space Sci. Rev., 155, 147-175, https://doi.org/10.1007/s11214-010-9663-x, 2010.

McLeod, M. G.: Spatial and temporal power spectra of the geomagnetic field, J. Geophys. Res., 101, 2745-2763, 1996.

Mound, J. and Buffett, B.: Detection of a gravitational oscillation in length-of-day, Earth Planet. Sci. Lett., 243, 383-389, 2006.

Olsen, N. and Mandea, M.: Investigation of a secular variation impulse using satellite data: The 2003 geomagnetic jerk, Earth Planet. Sci. Lett., 255, 94-105, 2007.

Olsen, N., Mandea, M., Sabaka, T. J., and Tøffner-Clausen, L.: CHAOS-2-A geomagnetic field model derived from one decade of continuous satellite data, Geophys. J. Int., 179, 1477-1487, https://doi.org/10.1111/j.1365-246X.2009.04386.x, 2009.

Ou, J., Du, A., and Finlay, C. C.: Quasi-biennial oscillations in the geomagnetic field: Their global characteristics and origin, J. Geophys. Res.-Space Phys., 122, 5043-5058, https://doi.org/10.1002/2016JA023292, 2017.

Paige, R. and Trindade, A.: The Hodrick-Prescott Filter: A special case of penalized spline smoothing, Elec. J. Stat., 4, 856-874, https://doi.org/10.1214/10-EJS570, 2010.

Pinheiro, K. J., Jackson, A., and Finlay, C. C.: Measurements and uncertainties of the occurrence time of the 1969, 1978, 1991, and 1999 geomagnetic jerks, Geochem. Geophys. Geosyst., 12, Q10015, https://doi.org/10.1029/2011GC003706, 2011.

Qamili, E., De Santis, A., Isac, A., Mandea, M., Duka, B., and Simonyan, A.: Geomagnetic jerks as chaotic fluctuations of the Earth's magnetic field, Geochem. Geophys. Geosyst., 14, 839850, https://doi.org/10.1029/2012GC004398, 2013.
Roberts, P.H., Yu, Z., and Russell, C.: On the 60-year signal from the core, Geophys., Astrophys. Fluid Dyn., 101, 11-35, https://doi.org/10.1080/03091920601083820, 2007.

Silva, L., Jackson, L., and Mound, J.: Assessing the importance and expression of the 6 year geomagnetic oscillation., J. Geophys. Res., 117, B10101, https://doi.org/10.1029/2012JB009405, 2012.

Soare, A., Cucu, G., and Mandea Alexandrescu, M.: Historical geomagnetic measurements in Romania, Ann. Geofis., 41, 539-554, 1998.

Stefan, C., Dobrica, V., and Demetrescu, C.: Core surface subcentennial magnetic flux patches: characteristics and evolution, Earth Planet. Space, 69, 146, https://doi.org/10.1186/S40623017-0732-1, 2017.

Torta, J. M., Pavón-Carrasco, F. J., Marsal, S., and Finlay, C. C.: Evidence for a new geomagnetic jerk in 2014, Geophys. Res. Lett., 42, 7933-7940, https://doi.org/10.1002/2015GL065501, 2015.

Valach, F., Vaczyova, M., and Dolinski, P.: Geomagnetic repeat stations network over the territory of Slovakia, Contrib. Geophys. Geodesy, 34, 21-42, 2004.

Verbanac, G., Lühr, H., Korte, M., and Mandea, M.: Contributions of the external field to the observatory annual means and a proposal for their corrections, Earth Planet. Space, 59, 1-7, 2007.

Wardinski, I. and Holme, R.: Signal from noise in geomagnetic field modeling: denoising data for secular variation studies, Geophys. J. Int., 185, 653-662, https://doi.org/10.1111/j.1365246X.2011.04988.x, 2011.

Yukutake, T. and Cain, J. C.: Solar cycle variations of the first degree spherical harmonic components of the geomagnetic field, J. Geomagn. Geoelectr., 11, 509-544, 1979.

Zatman, S. and Bloxham, J.: Torsional oscillations and the magnetic field within the Earth's core, Nature, 388, 760-763, 1997. 